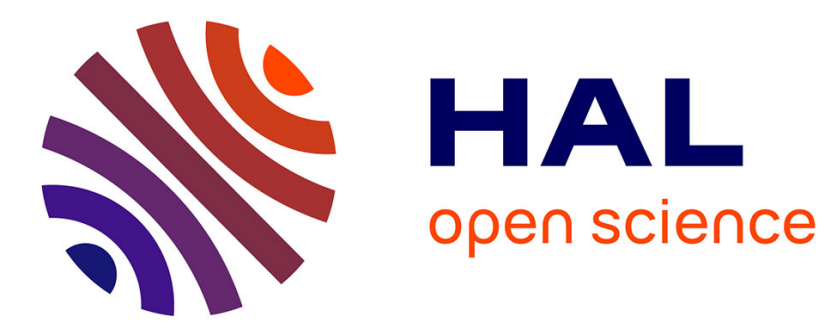

\title{
Le chasseur et son fusil en Haute-Provence
}

Nicolas Govoroff, Arundhati Virmani

\section{To cite this version:}

Nicolas Govoroff, Arundhati Virmani. Le chasseur et son fusil en Haute-Provence. Technical Choices: Transformations in Material Cultures since the Neolithic, P. Lemonnier (ed.), London \& New York: Routledge 1993: 227-237, 2010, pp.509-527. 10.4000/tc.5018 . hal-02527451

\section{HAL Id: hal-02527451 \\ https://hal.science/hal-02527451}

Submitted on 8 Apr 2020

HAL is a multi-disciplinary open access archive for the deposit and dissemination of scientific research documents, whether they are published or not. The documents may come from teaching and research institutions in France or abroad, or from public or private research centers.
L'archive ouverte pluridisciplinaire HAL, est destinée au dépôt et à la diffusion de documents scientifiques de niveau recherche, publiés ou non, émanant des établissements d'enseignement et de recherche français ou étrangers, des laboratoires publics ou privés. 


\section{Le chasseur et son fusil en Haute-Provence}

\section{Nicolas Govoroff}

\section{(2) OpenEdition}

\section{Journals}

Édition électronique

URL : http://journals.openedition.org/tc/5018

DOI : $10.4000 /$ tc. 5018

ISSN : 1952-420X

\section{Éditeur}

Éditions de l'EHESS

\section{Édition imprimée}

Date de publication : 30 juin 2010

Pagination : 509-527

ISSN : 0248-6016

\section{Référence électronique}

Nicolas Govoroff, "Le chasseur et son fusil en Haute-Provence », Techniques \& Culture [En ligne], 54-55 | 2010, mis en ligne le 30 juin 2013, consulté le 02 mai 2019. URL : http:// journals.openedition.org/tc/5018; DOI : 10.4000/tc.5018 


\section{LE CHASSEUR ET SON FUSIL EN HAUTE-PROVENCE}

in Technical Choices: Transformations in Material Cultures since the Neolithic,

P. Lemonnier (ed.), London \& New York : Routledge 1993 : 227-237

Depuis que les chasseurs se sont mis à utiliser les armes à feu, les anthropologues semblent avoir perdu tout intérêt pour les armes. Et les quelques rares chercheurs qui font exception se sont principalement concentrés sur la question de l'efficacité technique, faisant la comparaison entre les vertus des armes traditionnelles (arcs et flèches, épées, sarbacanes...) et les armes à feu manufacturées ${ }^{1}$. Mais dès que les premières disparaissent - que ce soit matériellement ou de la mémoire des informateurs les plus âgés -, les secondes ne sont plus évoquées, même dans les études folklorisantes ou «locales». Elles sont au mieux considérées comme allant de soi et écartées en quelques lignes. Plus fréquemment, elles sont éliminées de l'enquête anthropologique parce qu'elles ne sont pas produites ou décorées par les usagers eux-mêmes, comme si elles ne pouvaient pas être matière à investissement culturel. Pourtant, comme Bromberger le remarque (1979 : 105), ces objets « comme les mots, sont porteurs d'informations ».

Cet article vise à montrer que les armes à feu et les munitions peuvent être les vecteurs d'une signification méta-technique qui renvoie à l'organisation sociale.

Les données viennent de l'ethnographie du sud-est de la France, et plus spécifiquement de la haute vallée du Verdon dans la partie alpine de la Provence, près de la frontière italienne. Cette région montagneuse est presque entièrement couverte de forêts qui s'étendent jusqu'à une altitude d'environ 1800 mètres et relayées ensuite par des pâturages d'altitude culminant à 3000 mètres. Le territoire non agricole représente 97 \% de la surface totale. La population est rare, âgée et dispersée en petits villages. Comme dans le reste du sud de la France, la chasse - qui n'est pas une activité de subsistance - est presque une institution, regroupant les hommes dans des associations locales, les sociétés 
de chasse au sein desquelles, Chamboredon l'a montré, les individus expriment leur « vraie citoyenneté » (1982 : 240). Les chasseurs la revendiquent comme une citoyenneté égalitaire, mais l'analyse de leurs armes indique que l'organisation sociale des groupes de chasse est plus complexe que leurs membres ne le prétendent.

La pratique de la chasse en France est soumise à un examen national, le permis de chasser, qui peut être obtenu dès l'âge de seize ans. Pourtant, de nombreux chasseurs commencent leur vie cynégétique plus tôt. Dans la vallée du Verdon, les garçons armés de carabines à air comprimé font souvent leurs premières sorties à l'âge de dix ou onze ans. La phase initiale d'apprentissage du chasseur a lieu dans le village même. Au début, ses cibles favorites sont des boîtes de conserve, des bouteilles et parfois les lampadaires du village. Peu après, il se tourne vers le « vrai » gibier, tels que les lézards ou les chats errants. Quelques mois plus tard, il se risque - pas trop loin - dans les champs alentours pour chasser des petits oiseaux (ortolan rouge, Antheus capestris; le phaéton à brins rouges, Phoenicurus ochruros; le bec-croisé des sapins, Loxia curvirostra, etc.) localement appelés simplement « petits ». Ces oiseaux, protégés par la loi et jamais tués par les adultes, sont le gibier spécifique des enfants.

Un ou deux ans plus tard, le jeune garçon accompagnera son grand-père à un poste de chasse éloigné du village. Là, sous la surveillance du vieil homme, il apprend à manier des armes à feu en sûreté (Bromberger et Dufour 1982). Le fusil utilisé est d'un très petit calibre de 9 millimètres, parfois 14 millimètres. Les chasseurs le dénomment par le diamètre intérieur du canon, précédé par un article qui indique son genre féminin. On dit « une neuf millimètres » ou « une quatorze millimètres ». Le genre, féminin, du fusil de chasse est dû au fait que la loi française considère cette arme à feu comme une petite carabine, parce qu'il utilise des cartouches à percussion centrale. Pourtant, les plombs tirés sont semblables à ceux d'un fusil de chasse.

À cette étape, la chasse consiste à tirer par les ouvertures d'un abri sur les oiseaux proches. Le gibier principal est constitué de grives (Turdus pilaris, Turdus visvicorus viscivorus, Turdus ericetorym philomelos et Turdus musicus musicus), de merles noirs (Turdus merula merula et Turdus torquatus), de geais (Garulus glandarus) et de temps à autre de pigeons sauvages (Columba palumbus).

Vers l'âge de 14 ans, un garçon quitte véritablement l'abri pour tenter sa chance sur un plus gros gibier, tels les lièvres (Lepus europaeus et Lepus timidus), les faisans (Phasianus colchicus), les perdrix et les pigeons. Il chasse seul, loin de son village, avec la carabine de chasse de calibre de 14 millimètres mentionnée précédemment. Cette arme ne permet pas au jeune chasseur de poursuivre toutes sortes de gibier. Ainsi par exemple, le sanglier sauvage (Sus scrofa) ne peut être abattu car il n'y a aucune munition appropriée à ce fusil sur le marché français. Un chasseur débutant est par conséquent techniquement limité au petit gibier ${ }^{2}$.

À l'âge de 15 ans, un an avant de se présenter à l'examen officiel de chasse, le garçon change une nouvelle fois d'arme pour adopter ce qui est considéré comme son premier « vrai » fusil de chasse. La distinction avec les armes à feu précédentes est essentiellement d'ordre légal. L'équipement du garçon passe littéralement de la catégorie «petite carabine » à « fusil de chasse ». Pour un anthropologue, il n'y a pas de différences techniques majeures entre les deux types de fusils. La plus remarquable serait le calibre. Traditionnellement, les Français le définissent ainsi : « Le nombre de plombs sphériques (de même calibre que le canon) qui peut être produit à partir d'une livre de plomb pur - un ancien poids qui équivaut 0,4895 kg » (Noblet 1988: 101). En pratique, on peut 
facilement identifier les divers calibres par le diamètre du canon ou par la dimension de la munition. Pour un chasseur cependant, le fusil de chasse a plusieurs avantages sur une carabine; en premier lieu, les cartouches contiennent plus de poudre, «la portée utile $»^{3}$ augmente en même temps que l'efficacité du chasseur; et en second lieu, on peut trouver les munitions adéquates à chaque type de gibier.

En français, cette arme s'appelle « un fusil »; le terme est masculin et, comme dans le cas précédent, il est identifié par un nombre qui indique son calibre, mais sans unité de mesure. Les fusils les plus ordinaires sont « le 20 », « le 16 » et « le $12 »$. Presque tous les adolescents ont des fusils de chasse de calibre 20.

On peut se demander pourquoi l'activité illégale des garçons n'a pas été interdite par les adultes ou par le garde-chasse de l'État. En fait, cette activité n'est pas seulement tolérée, elle est encouragée par d'autres chasseurs qui la considèrent comme une phase nécessaire d'apprentissage tant du point de vue technique (usage des armes à feu) que de celui qu'on pourrait appeler "écologique » (connaissance du territoire de chasse, des habitudes du gibier...). Ce genre de braconnage est donc considéré comme légitime, bien qu'illégal, pour reprendre la distinction de Traimond (1984). Les gardes-chasse se montrent également tolérants car, comme certains l'avouent, ils ont commencé leur « carrière » de la même façon (Govoroff 1985 : 78). Mais cette « compréhension » des gardes s'applique seulement aux jeunes du lieu, qui essaient néanmoins de les éviter.

Désormais, le garçon participe à la chasse en groupe avec les adultes qui passent la plupart de leur temps à pister les animaux les plus « nobles » : le sanglier sauvage, le chevreuil (Capreolus capreolus) et, dans quelques endroits, le chamois (Rupicapra rupicapra).

Quoique le jeune soit admis parmi les « grands », il n'est pas encore considéré comme adulte, et son équipement est toujours moins efficace. Pendant cette dernière saison de chasse illégale, il apprend à la fois les règles de base de chasse en groupe, qui consistent essentiellement à ne pas tirer dans la direction des gens ou des chiens de chasse et à rester immobile, silencieux et inodore (sans fumer ni se soulager par exemple) pendant plusieurs heures à son poste ${ }^{4}$.

Enfin, 16 ans! L'heure de passer l'examen de chasse national. La grande majorité le réussit. Encore une fois, le garçon change de fusil. Pour la première fois, il chassera avec le même type d'arme que ses aînés, le fusil de calibre 12. En plus, il échangera aussi son arme à feu à un seul canon et un seul coup pour un canon double. Le changement de statut légal et d'arme coïncide aussi avec un changement de statut social dans le groupe. Le garçon est devenu un adulte. Dans la haute vallée du Verdon, cette transition est illustrée par deux termes différents dans le dialecte local. Habituellement, on appelle un garçon soit par son nom soit par le terme générique « minot », qui signifie " petit » pour les hommes, mais à partir de maintenant le chasseur sera appelé « jeune » (Bromberger et al. 1981 : 51). Et il reste « jeune » jusqu'au jour de son mariage. Si un homme ne se marie pas, cette désignation ne sera pas utilisée au-delà de l'âge de 40 ans environ. Le groupe d'âge « jeune » équivaut donc implicitement à « mari potentiel ». Ainsi, à 16 ans, la sexualité du garçon est culturellement affirmée et reconnue. Au même moment, alors que le chasseur passe de l'illégalité à la légalité, il entre dans l'âge d'homme adulte mais, pour ainsi dire, sur la pointe des pieds. Si la sexualité réelle du jeune homme n'est jamais discutée, l'affirmation sociale de cette activité vient seulement après sa première grande mise à mort, préférablement d'un sanglier ou d'un chamois ${ }^{5}$; ou, pour être précis, après la castration ${ }^{6}$ de l'animal mort s'il a eu la chance d'avoir abattu un mâle. De retour au village, où le partage du gibier se fait toujours, il sera félicité par tous, hommes 
et femmes. Il aura aussi à supporter des coups de poing affectueux et des tapes de ses camarades de chasse, et, plus surprenant, certaines caresses qui, dans cette partie de la France, sont généralement considérées comme ayant une connotation féminine ou homosexuelle. Les caresses sont faites sur les fesses et la partie supérieure de la cuisse, en fait, tout autour des parties génitales du chasseur - celles-ci restant taboues - « comme si » l'émasculation du sanglier avait transféré la virilité de l'animal au chasseur qui, à cette occasion, est appelé « tueur». La clause « comme si », impliquant une relation de causalité, est partiellement confirmée par la remarque d'un vieux chasseur: « Alors? Tu t'es dépucelé? » Le simple fait d'avoir touché le sanglier est en lui-même, mais à un moindre degré, un acte masculinisant, comme l'illustre la remarque suivante adressée à un garçon de trois ou quatre ans qui agrippait les poils d'un sanglier mort: "Ça en fera un homme! ». Le lien entre l'examen de chasse, le choix de l'arme et l'entrée dans le statut d'homme n'est pas qu'une simple corrélation.

La première mise à mort d'un sanglier peut arriver aussi bien en un jour qu'après plusieurs années. Dans l'intervalle, le chasseur a pu changer d'arme à feu, remplaçant souvent le fusil à canon double par un fusil de chasse automatique de calibre 12 à canon simple. Pourtant, l'engouement pour ce genre d'arme diminue aujourd'hui et les gens reviennent au canon double, plus classique.

Dans la vie d'un homme, la prochaine étape importante est sa majorité civile qui, en France, est fixée à 18 ans. Le jeune chasseur peut maintenant acheter légalement son propre équipement. Auparavant, il l'empruntait, le recevait comme cadeau ou l'achetait par l'intermédiaire d'un parent. La loi française lui permet aussi d'acheter et d'utiliser une carabine de gros calibre, considérée comme l'arme ultime pour le gros gibier. La différence entre une carabine et un fusil de chasse réside essentiellement dans le fait que la première ne tire qu'une seule balle sur une longue distance. Avec un tel instrument, la portée de tir utile augmente considérablement, passant approximativement de 50 mètres avec un fusil de chasse à quelque 200 mètres avec une carabine. En fait, les calibres les plus recherchés dans cette région sont le $7 \times 64$ français, le Winchester .270 et le Remington .280 anglo-saxons.

L'usage de cette carabine est, comme pour toutes les autres armes, soumis à l'approbation du groupe, et n'importe qui ne peut l'obtenir. Il est limité aux chasseurs réputés endurcis, l'expérience se résumant au nombre de sangliers ou de chamois tués, bien qu'aucun minimum défini ne soit établi. Il semblerait qu'il varie d'un individu à un autre selon les conditions dans lesquelles les animaux ont été abattus. Étaient-ils déjà blessés? Étaient-ils touchés aux endroits vitaux? Est-ce que le tir du chasseur stoppe souvent les animaux touchés? L'acquisition d'une carabine est toujours suggérée par un membre déjà expérimenté du groupe. J'ai été témoin de quelques cas où un individu a pris ce type de liberté sans approbation préalable. En général, on se moque de lui et sa compétence est mise en question: "Tu ne peux même pas tirer droit! » résume la moquerie en quelques mots. Mais parfois le contrôle social peut être plus stricte, et les chasseurs refuseront d'être " postés » à portée de carabine de l'autre, pour des raisons évidentes de sécurité. Pourtant, le chasseur prétentieux ne sera pas rejeté du groupe. Il sera posté loin des autres, souvent aux endroits où aucun gros gibier ne risque de passer. Je suppose, même si je n'ai pas eu l'occasion de tester cette hypothèse, que cette situation dure tant que le chasseur n'a pas démontré son habileté, ce qui naturellement est difficile aux emplacements qui lui sont désignés; mais cela peut arriver puisque, par définition, la fuite d'un animal n'est pas complètement prévisible. Quand un tel 
événement se produit, cela permet aux chasseurs de soutenir que tous les postes sont équivalents même s'il est évident qu'ils ne le sont pas.

Certains chasseurs ont depuis peu adopté une arme appelée drilling. Cette arme combine les avantages techniques d'un fusil à canon double juxtaposé, auquel elle ressemble par la forme, et ceux d'une carabine car elle possède aussi un canon de fusil à un coup sous les deux autres. Cet hybride permet à l'usager de sélectionner la munition appropriée au dernier moment. Chasser avec un drilling obéit aux mêmes règles sociales qu'avec une carabine.

À ce stade de sa vie, un chasseur possède généralement au moins deux armes différentes: un fusil de calibre 12, principalement mais pas exclusivement pour le petit gibier, et une carabine pour le gros gibier. Mais avec le temps qui passe, et la vieillesse qui approche, le chasseur abandonne la carabine pour une arme de calibre 20 ou souvent de calibre 16. La raison invoquée est que les plus petits fusils sont moins fatigants à porter et à viser puisqu'ils sont plus légers et ont moins de recul. La conséquence immédiate de ce changement est la réduction de la portée de tir du chasseur et donc le déclin de sa performance. Une autre conséquence est la diminution du nombre de postes qu'on peut lui donner, puisque sa mobilité limitée à cause de l'âge lui interdit des approches longues. Le vieil homme abandonne la chasse en groupe et la chasse au gros gibier quand son incapacité est devenue presque totale. Toutefois, sa vie de chasseur peut continuer s'il est encore capable de parcourir les quelques mètres qui séparent sa voiture d'un abri de chasse où il passera la plupart de son temps à tirer sur les grives en compagnie d'un petit-fils. Il utilisera alors une petite carabine de 14 ou de 9 millimètres. Ainsi, le cycle de vie d'un chasseur dessine une figure complète qui le reconduit là où il débuta quelque soixante ans plus tôt, où il usait de la même technique, de la même arme et pour le même gibier.

Jusqu'ici, nous avons seulement traité de l'équipement des hommes, puisque la chasse est traditionnellement une activité masculine et virile. Pourtant, quelques femmes aussi prennent part à la chasse de groupe aux côtés de leurs maris. Pour elles, les chasseurs recommandent des fusils de calibre 20, plus légers mais moins précis, confirmant ici aussi, au moins d'un point de vue normatif, l'affirmation de Tabet que « [dans] les activités qui requièrent un outillage d'une certaine complexité [les femmes] doivent se contenter d'outils plus rudimentaires et souvent moins spécialisés que ceux employés par les hommes qui exercent les mêmes activités dans la même société » (1979: 13).

Comparée à celle d'un homme, la vie cynégétique d'une femme ne passe pas par les différentes phases décrites ci-dessus. Elles entrent dans le « cycle » à l'étape du jeune chasseur, et elles y restent, ne changeant jamais d'armes même si elles connaissent des succès à la chasse. Dans ce cas, les histoires de chasse racontent toujours que les plus gros animaux sont tués par les femmes ${ }^{7}$. La position qu'elles occupent et leur manque de progrès sont expliqués par le fait que, comme les adolescents, les femmes sont connues pour être des chasseurs passionnés mais, ainsi que l'explique un garde-chasse, contrairement aux jeunes hommes, « elles ne comprennent rien » comme si le temps et l'expérience leur échappaient.

Un examen minutieux de l'équipement des femmes montre qu'elles ne chassent pas toutes avec les armes recommandées, certaines préférant des fusils de calibre 12 . Comme dans le cas d'un jeune-homme qui utilise un fusil de gros calibre, le contrôle social s'opérera par la moquerie. Cette fois, pourtant, son objet n'est pas le chasseur - une femme dans ce cas- mais le fusil lui-même, qui est comparé à une sarbacane ou à un tromblon, et si c'est une arme semi-automatique, à une pompe à vélo, surtout après 
un échec. Dans la Provence alpine, les femmes chasseurs sont considérées comme des "presque chasseurs», ressemblant aux garçons de 15 ans. La différence pourtant est que ces derniers, de fait, sont « de futurs chasseurs».

Mais revenons à un homme au sommet de sa carrière, quand il manie tout à la fois une carabine et un fusil. Quelques hommes utilisent des fusils de calibre 20 plutôt que de calibre 12, même s'ils en possèdent également un en général. Quand on leur demande pourquoi ils utilisent un si petit calibre, ils répondent fièrement: "C'est un fusil de femme » et expliquent ensuite que les bons chasseurs - c'est-à-dire les meilleurs! - font toujours ainsi. Ce statut de meilleur chasseur est autoproclamé et vraisemblablement soumis à l'approbation tacite du groupe. Ayant déjà démontré sa valeur en tant que chasseur, et donc en tant qu'homme, il peut sans danger se tourner vers des armes moins efficaces; sa connaissance du terrain et des habitudes du gibier ajoutée à la précision de son objectif compensent la puissance plus faible du fusil. On peut dire qu'à ce moment de sa vie, le meilleur chasseur autoproclamé est tout autant à la recherche de prestige que de gibier, bien que cette distinction ne soit qu'en partie valable, les deux aspects se déterminant mutuellement. Ainsi, paradoxalement, les plus «virils» des individus proclament leur masculinité en utilisant des fusils à connotation féminine.

Dans chaque catégorie d'armes de chasse décrites, de nombreux modèles existent, parmi lesquels beaucoup sont en vente au supermarché local. Comment les chasseurs choisissent-ils leur équipement parmi la grande variété présentée? La plupart des villageois de la haute vallée du Verdon ont de petits revenus, et le premier paramètre influençant le choix est bien sûr le prix. Au total, le fusil le plus communément acheté coûte entre 3000 et 4000 francs français (entre 450 et 600 euros), ce qui représente [dans les années 1980] plus ou moins $65 \%$ du salaire mensuel. Pour une carabine, le prix peut atteindre 75 à 80 \% du même revenu et pour un drilling, un chasseur doit consacrer au moins deux mois de salaire, ce qui explique en partie sa lente diffusion.

Le second ensemble de paramètres concerne l'adaptation du fusil à la morphologie du chasseur. Un fusil peut être modifié de différentes façons pour convenir à son utilisateur. Dans le sud de la France, cela n'est jamais fait à l'exception de rares cas de rallongement de la crosse avec des blocs de plastique anti-recul. En général, les chasseurs s'adaptent eux-mêmes à l'arme, qui agit comme une sorte de «mémoire inerte » de la bonne position, cette dernière étant déterminée scientifiquement (c'est-à-dire ergonomiquement) par le fabricant ${ }^{8}$. Cette mémoire inerte renforce ce que Mauss (1983) a appelé «les techniques du corps » c'est-à-dire la façon dont le corps humain est en lui-même un outil que chaque culture mobilise différemment, tout en répondant aux mêmes besoins (c'est-à-dire marcher, nager, etc.). Il n'était cependant pas entré dans les implications des « techniques du corps » liées à des techniques instrumentales. L'idée avancée ici est qu'il y a une relation réciproque entre ces deux types de techniques, bien que le présent article se concentre principalement sur les premières, puisque les chasseurs ne produisent pas leurs propres armes.

Le troisième et dernier ensemble de paramètres est, à mon avis, mineur. Il inclut les détails techniques de différentes sortes, par exemple: est-ce que la bande de visée est ventilée ou non, ou est-ce que la mire est composée d'un point de bronze ou d'une perle rouge phosphorescente brillante?

Les munitions sont souvent considérées comme la simple contrepartie technique du fusil et sont rarement mentionnées. Mais une arme à feu n'abat pas le gibier, ce sont 
les projectiles qui le font. Et leur importance est telle qu'ils constituent le fil sous-jacent préludant au choix d'un fusil. Et là aussi, les usages sociaux qui leur sont liés vont au-delà de la fonction purement technique pour laquelle ces munitions sont produites.

À chaque catégorie d'arme de chasse correspond une catégorie de munition, qui, à son tour, peut être divisée en de nombreuses sous-catégories définies par des critères euxmêmes tout aussi nombreux. Le plus évident est la taille des projectiles. Elle est censée varier en fonction du gibier chassé. Selon les chasseurs, la grive exige des cartouches numéro 8 (plomb de 2,25 mm); le lièvre, des cartouches numéro $2(3,75 \mathrm{~mm})^{9}$; le chevreuil, le chamois et le sanglier exigent des balles. Sur le terrain, les chasseurs sont aussi autorisés à tirer le sanglier avec des chevrotines ${ }^{10}$. Bien que la variété des munitions rende cette adaptation possible, aucune distinction n'est faite entre deux animaux différents de la même espèce, notamment en taille, et cela, alors même que tous les manuels de chasse le conseillent. Dans ce cas, le calibre des projectiles choisis semble être davantage lié à l'espèce animale chassée qu'à sa taille - ceci révélant une connexion possible entre choix technique et classification taxinomique. Les chevreuils ne sont par exemple jamais chassés avec des cartouches numéro 2, même si cela est autorisé. Un principe identique s'applique au suicide pour lequel les chasseurs choisissent toujours des munitions de gros calibre destinées au grand gibier alors qu'à bout portant, des munitions pour oiseau seraient suffisantes.

Pour chaque type de munition, plusieurs modèles diffèrent en calibre, taille, forme, couleur, composition du métal, quantité de poudre et ainsi de suite. Le choix effectué par chaque chasseur sert l'objectif évident de décliner la mise à mort mais a aussi pour effet « d'imprimer, à distance, les marques personnelles du tueur sur sa proie » (Ingold 1980 : 157). Lors d'une chasse collective, la mise à mort est généralement attribuée à la dernière personne qui a tiré sur l'animal, sauf quand il s'agit simplement de l'achever. Pourtant, l'attribution définitive n'est véritablement révélée qu'au moment du dépeçage où les projectiles sont analysés et le plus létal identifié. Si des doutes persistent, on demande aux tueurs potentiels de fournir un rapport précis de leurs actions. Un débat s'ensuit jusqu'à ce qu'un consensus soit établi par le reste du groupe et approuvé par son chef. Dans ces cas rares, les éléments autres que balistiques sont pris en compte, principalement les statuts respectifs des chasseurs concernés, en accord avec le principe que le prestige va au prestige. Bien sûr, ces éléments ne sont jamais présentés ouvertement. L'enjeu ici est seulement le prestige, puisque tous les chasseurs reçoivent la même quantité de viande. Parce que ce « prestige » est parfois concrétisé par un trophée, j’avancerai l'argument opposé à celui de Fabre-Vassas (1982 : 377), que la mise à mort elle-même et la castration qui suit sont déjà constitutives du partage et peuvent être considérées comme la récompense revenant de plein droit au tueur en contrepartie de la perte relative que représente la viande partagée, puisqu'il ne ramènera chez lui qu'une petite partie du gibier.

Quand quelqu'un emprunte soit une arme soit une munition, le gibier éventuellement tué n'est pas attribué au prêteur, comme cela se passe dans certaines cultures (Ingold 1980). Néanmoins, une relation amicale est instituée - souvent sans que le reste du groupe le sache - entre les deux protagonistes. Elle est explicitement activée par une phrase presque rituelle telle que « Ton fusil (ou ta cartouche) m’a porté chance ». On s'en souviendra et on le rappellera bien après l'événement. Le prêteur est ainsi gratifié, pour partie, du mérite de la mise à mort, même si celle-ci n'est pas portée à son compte.

Les cartouches vides sont aussi d'une certaine utilité. Elles sont fréquemment enfilées sur le bout d'une branche à l'endroit où le gros gibier a été tué. En combinaison avec 
les toponymes, elles servent de marqueurs et permettent de localiser précisément où se placer pendant une chasse. De plus, puisque les chemins précis du gibier peuvent changer légèrement d'une année à l'autre, la marque de la cartouche vide concrétise l'actuelle «meilleure position». Le changement est rarement important mais dans cette zone montagneuse boisée de la haute du vallée du Verdon, une différence de dix mètres peut permettre à un sanglier de passer sans être aperçu même s'il est probablement entendu.

Dans cette région, les chasseurs sont tous engagés dans une forme policée de compétition. Exposer les cartouches vides est une façon d'entretenir l'émulation, puisque la plupart des gens peuvent identifier avec quelque précision à la fois le chasseur et l'animal tué en comparant les caractéristiques de la cartouche et son emplacement. Par exemple, une cartouche de bronze à percussion centrale sur un terrain couvert de chênes indique que le chasseur utilisait un fusil et tirait soit sur un chevreuil soit sur un sanglier. Le calibre inscrit sur la cartouche rétrécit le champ de l'identification à une poignée de personnes. La zone où elle a été trouvée est aussi un indice du groupe de chasse auquel appartient le tueur, puisque la plupart des groupes ont, au sein de leur territoire, des terrains de chasse privilégiés ou préférés. Souvent, ces éléments suffisent à fournir une identification précise. Sinon, les événements récents seront pris en compte. L'état de la cartouche (degré d'oxydation, éventuelle persistance de l'odeur de poudre) renseignera quant à lui sur la date de la mise à mort du gibier et ainsi sur le chasseur chanceux.

Un processus semblable se rencontre avec les cartouches de fusil pour le petit gibier mais seulement pour des espèces prisées telles que le lièvre, la perdrix (Lyrurus tetrix) et la bécasse (Scopolax rusticola). La combinaison du calibre, de la fabrication, de la charge (normale, magnum ou super-magnum) et la taille de la base métallique, donne une idée approximative du propriétaire, mais rarement du gibier tué. Pour permettre aux autres d'identifier l'animal tué, et si la taille de la cartouche n'est pas suffisamment explicite, les chasseurs attachent quelques poils ou plumes de la proie abattue à l'objet. Le lien lui-même peut aider à déterminer l'auteur du signe ${ }^{11}$.

Ces signes représentent autant de lieux humanisés dans un environnement perçu comme sauvage et même « produit » comme tel (Bromberger et al. 1985 : 97). Pourtant, la durée de vie de ces signes - d'autres existent qui ne sont pas mentionnés ici - est brève et ne se prolonge jamais au-delà du dégel. L'humanisation par ce marquage du territoire doit donc être renouvelée chaque année.

Les usages sociaux des armes à feu ici ne sont en aucune façon traditionnels. Leur apparition remonte aux environs des années cinquante et coïncide avec l'accroissement du budget consacré à l'achat des armes dont le prix, lui, décroit avec l'industrialisation. Bien que les chasseurs ne soient pas impliqués dans le processus de fabrication, ils chargent leurs fusils de valeurs culturelles qui révèlent une organisation sociale et un fonctionnement dissimulés sous l'apparente égalité des membres du groupe. occultés par le groupe sur la base de l'égalité de ses membres.

Les anthropologues sont souvent confrontés au problème complexe de « choix techniques » apparemment effectués sans aucune raison évidente. Quand de tels cas surviennent, ils sont considérés avec raison comme des moyens privilégiés d'accéder à d'autres niveaux de la réalité sociale. Un des exemples les plus éclairants, à mon avis, est la remarque de Turnbull sur les techniques de chasse des pygmées Mbuti: «En ce qui concerne les Mbuti, il n'y a pas de raison environnementale pour que la moitié d'entre eux chassent au filet et l'autre à l'arc, bien qu'il y ait sans doute quelques raisons historiques » (1965 : 134). Qu'elle soit ou non mentionnée, la notion de « choix » 
est un artefact introduit dans les données pour rendre compte d'une situation x ou y. Geistdoerfer (1973 : 66) a montré que cette notion est à la croisée des concepts de LeroiGourhan de «tendance technique » et de « fait technique » $(1971,1973)$. Les « faits » sont des expressions ethnographiques attestées d'une «tendance », ici les armes à feu en général. Dans les cultures traditionnelles, chaque «tendance » est représentée par seulement quelques « faits ». Avec des outils industriels, fusils ou autres, précisément parce que les usagers n'ont aucun contrôle sur la production des « données », le choix technique peut être observé mais non postulé.

(Traduit de l'anglais par Arundhati Virmani) 


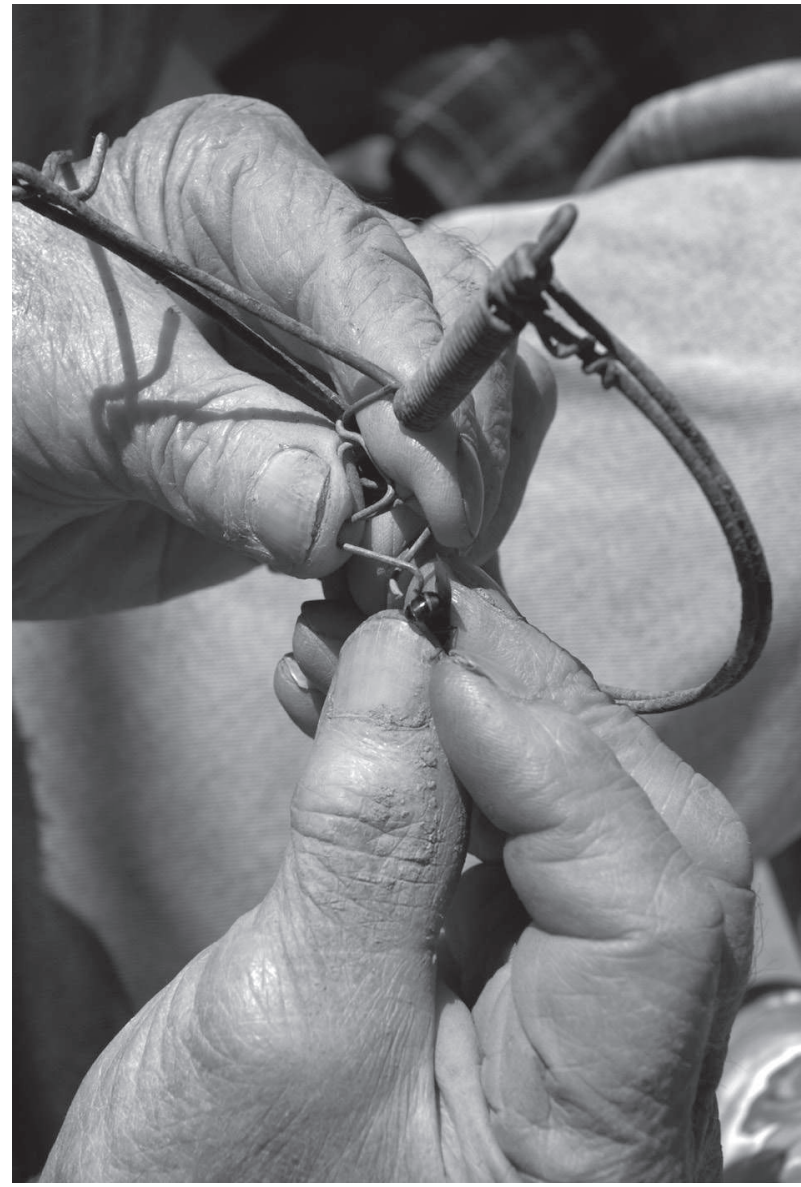

${ }^{\bullet} \mathrm{F}$. Joulian

Avec Nicolas, nous comparions les pratiques et usages de chasse et de braconnage en Haute et Basse-Provence et le rapport si étrange quill peut y avoir à capturer et manger le plus anthropophile des oiseaux, le rouge-gorge - le « rigaou » - attrapé à l'aide d'une tapette à rat et d'aludes précieusement conservées dans un bambou.

Cette prédation de proximité, à l'interface du champ et de la colline correspond à une pratique originale. Elle lie, plutôt que sépare, les savoirs naturalistes des conduites de prédation et de chasse analysées par Nicolas.

\section{Avec Nicolas Govoroff de la Provence à l'Afrique de l'Ouest}

« Zetou » montrant comment accrocher l'alude au « piège à rigaou » Carqueiranne, 2008

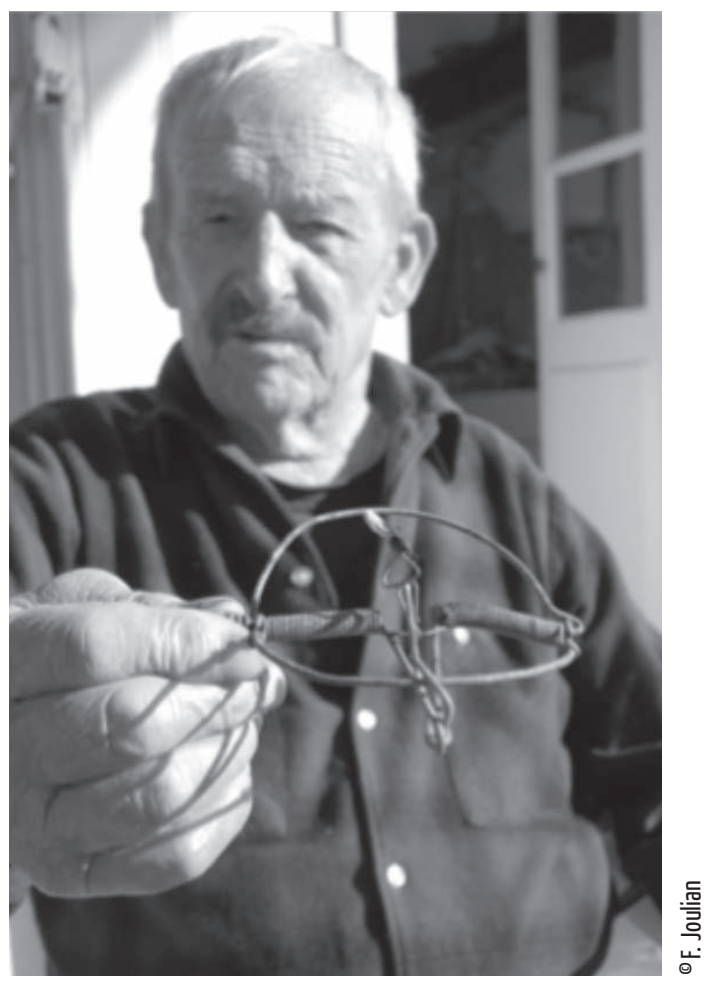




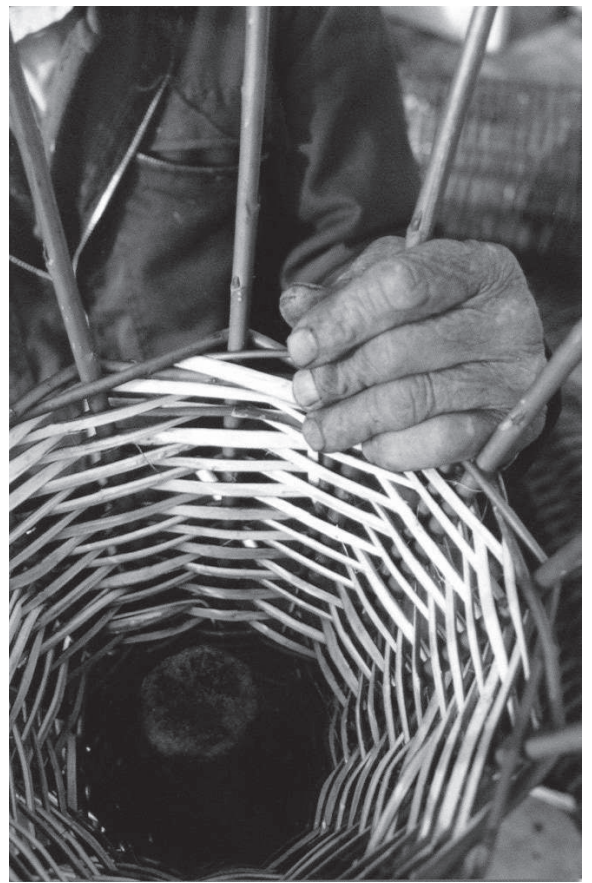

\section{Fabrication d'une nasse à lamproie, Dordogne, circa 1990}

Parmi les diverses réticulations de son travail sur la pêche dans le Sud-Ouest de la France, Nicolas Govoroff montrait l'importance d'une connaissance directe des matériaux, par exemple la torsion que l'on peut donner à l'osier lorsquion le travaille ou lorsque l'on s'intéresse à la transmission des savoir faire. \|l faisait saisir combien les situations de transmission au travers des générations peuvent être compliquées et paradoxales.

Pour aborder la question du langage dans les techniques, Nicolas Govoroff, se fondant sur une « observation participante », distinguait entre démarches silencieuses et descriptives et démarches verbalisées et explicatives. II fondait cette distinction sur des dizaines de chaines opératoires de fabrication, puis d'utilisation, quili avait engrangées au fil des années chez les pêcheurs à la lamproie de Dordogne.

Il s'interrogeait également sur la place de l'ethnologue et de ses mots dans le travail de restitution, à ses pairs, ou aux informateurs quill náavait de cesse de revoir; comme si le travail en détail, en immersion,

propre à la véritable ethnologie, permettait d'ouvrir aux questions

les plus générales qui soient - sociales, politiques et anthropologiques.

Comment mettre les choses en mots lorsque les signifiants ne sont que des gestes ou des expressions et que les objets (les nasses en l'occurrence) sont des métaphores des corps de femmes et renvoient à des interdits sur lesquels bute la pensée des acteurs?

(Govoroff, 2002, 2006).

Abuado Forest, Ghana, 2007

Nicolas montrant la « bouche » d'une nasse que nous avions découverte lors d'une prospection avec des chasseurs Fante dans une forêt classée.

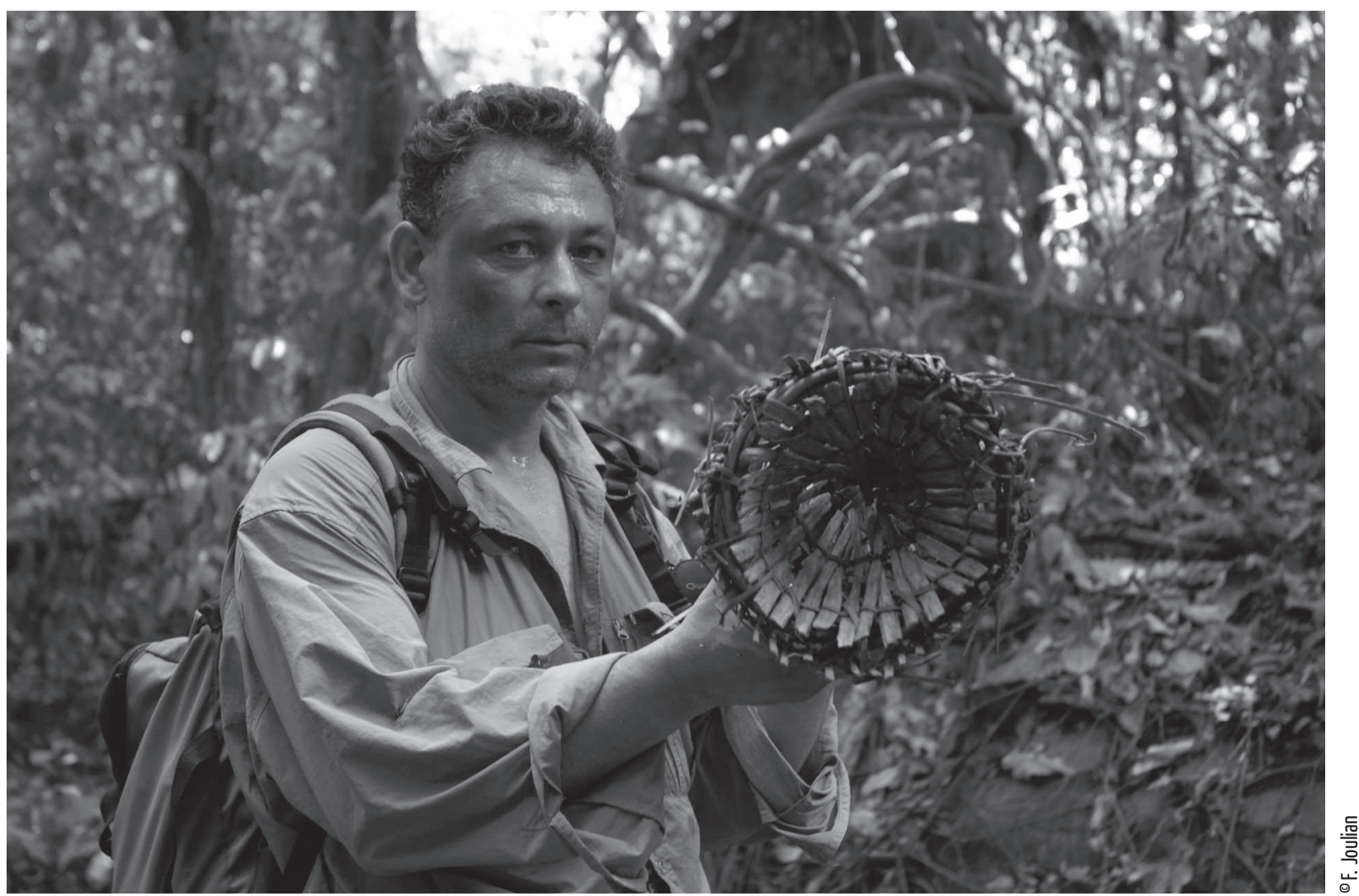




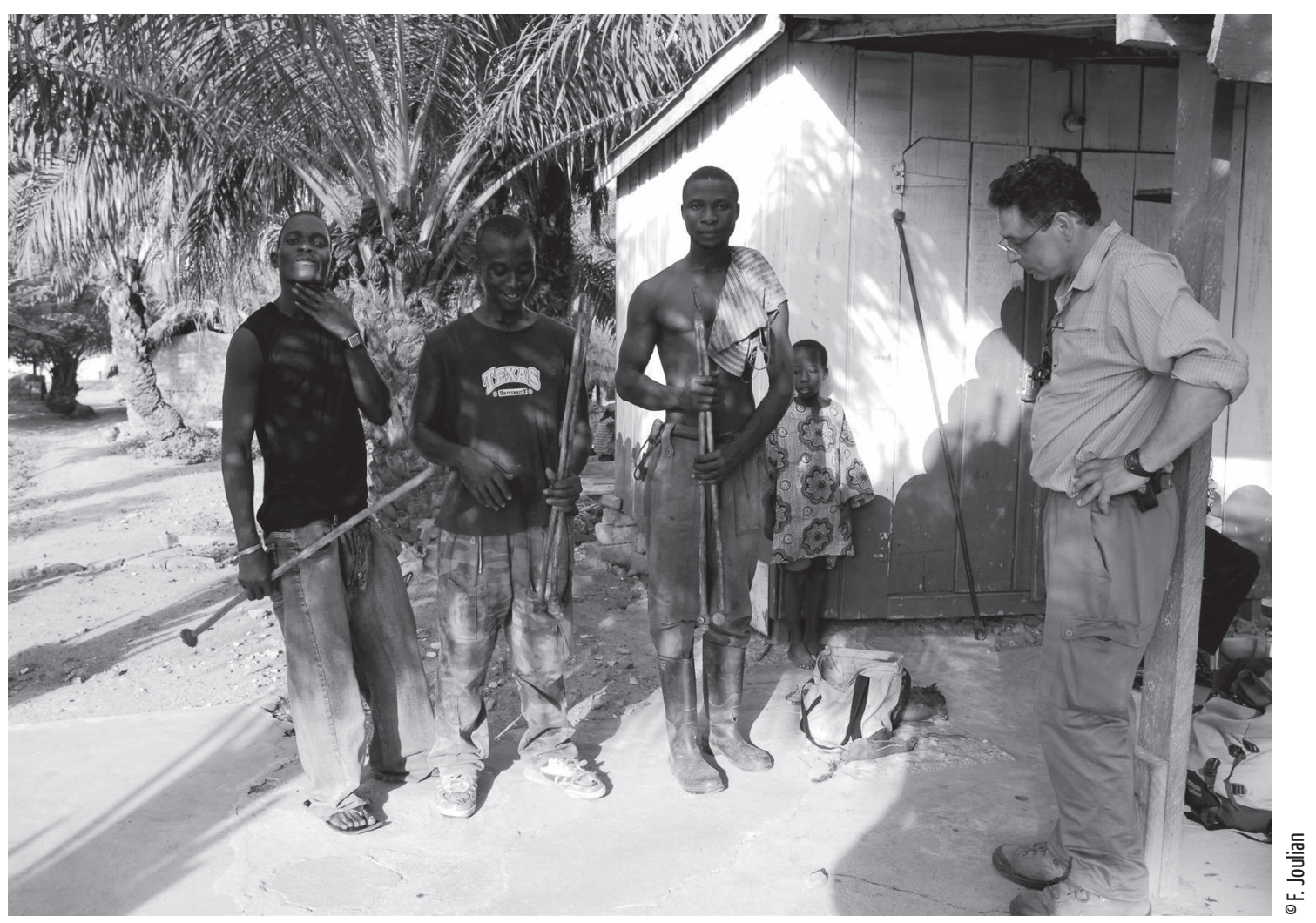

Chasse au bâton plombé avec chiens et lance-pierres

L'ethnologue observe, questionne prudemment, soupèse le gibier, s'amuse mais prend cette question de subsistance quotidienne très sérieusement. Ho, Ghana 2007

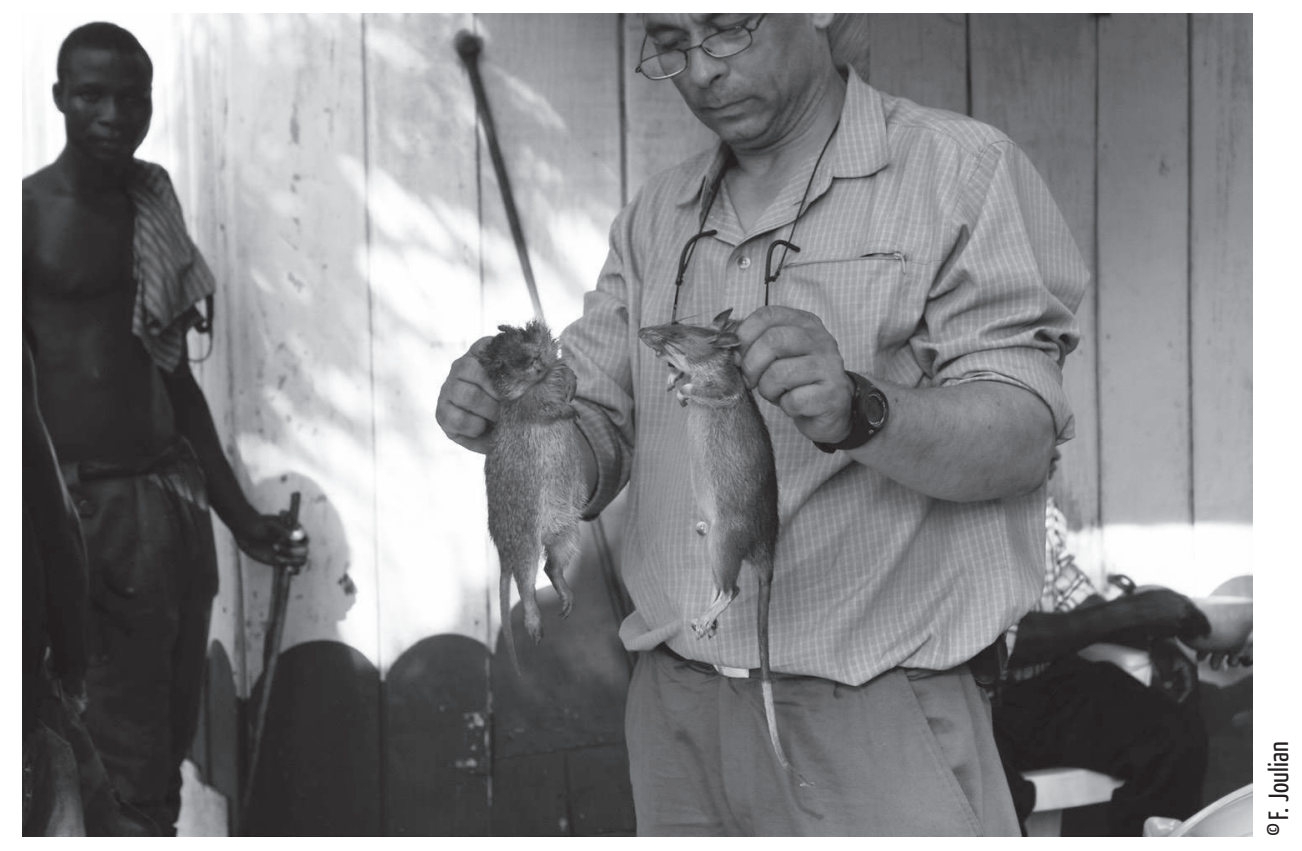



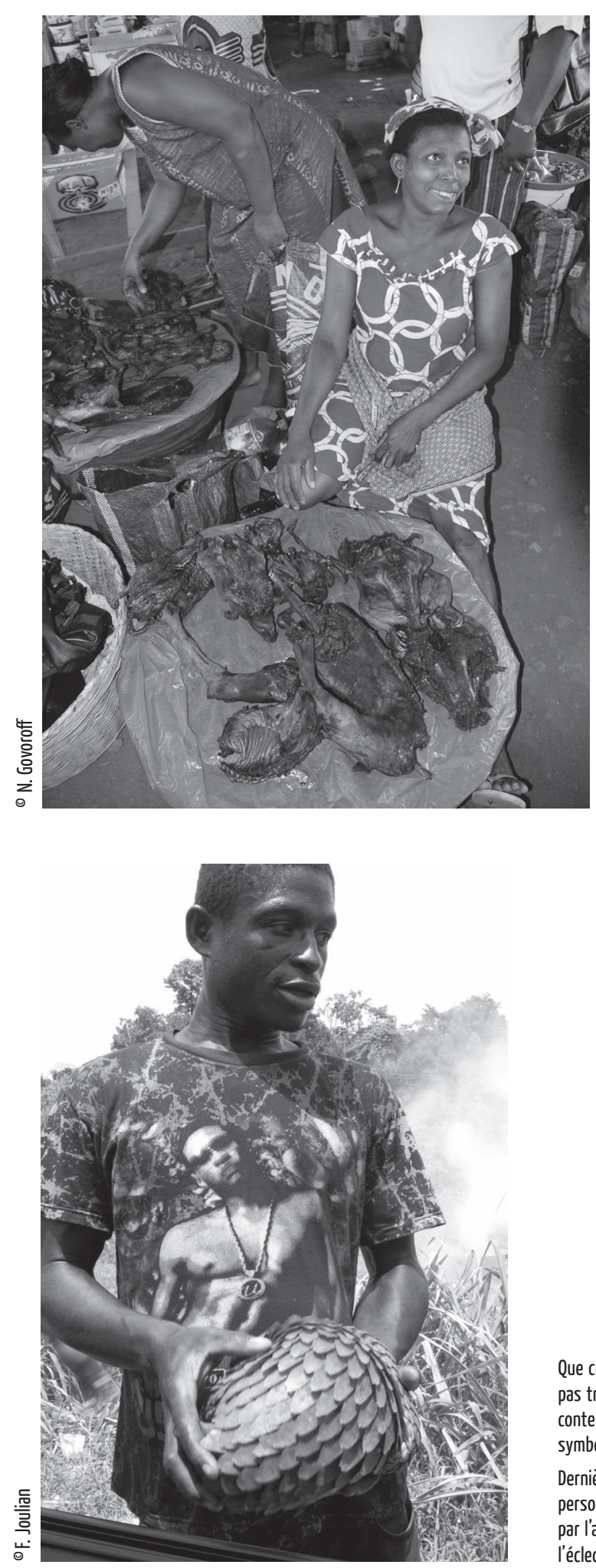

Deux filières du commerce de la viande de brousse

L'une instituée, conduite par des femmes sur un marché

dans la Volta Region, l'autre, opportuniste, pratiquée par des jeunes sur le bord d'une route passante dans le centre du Ghana, 2007.
Que ce soit en Afrique ou en Europe, les pratiques de chasse ne sont bien évidemment pas traditionnelles et intemporelles : elles doivent être comprises dans leurs formes contemporaines. Dans son article, Nicolas Govoroff, débusque « de la tradition » et « du symbolique » derrière un instrument manufacturé.

Dernièrement, il s'attachait à saisir les apports financiers de la chasse ou de la pêche pour des personnes à très faible revenu. Cette dimension économique, et disons peu prise en compte par l'anthropologie qui s'intéresse habituellement à des sujets plus « nobles », témoigne de l'éclectisme intellectuel nécessaire pour bien faire ce métier. 
Boabeng-Fiema, forêt classée du Ghana où les singes mones et colobes sont vénérés et protégés comme des ancêtres.

Ce qui en fait un des exemples les plus représentatifs d'une bonne conservation du patrimoine naturel et culturel au Ghana.

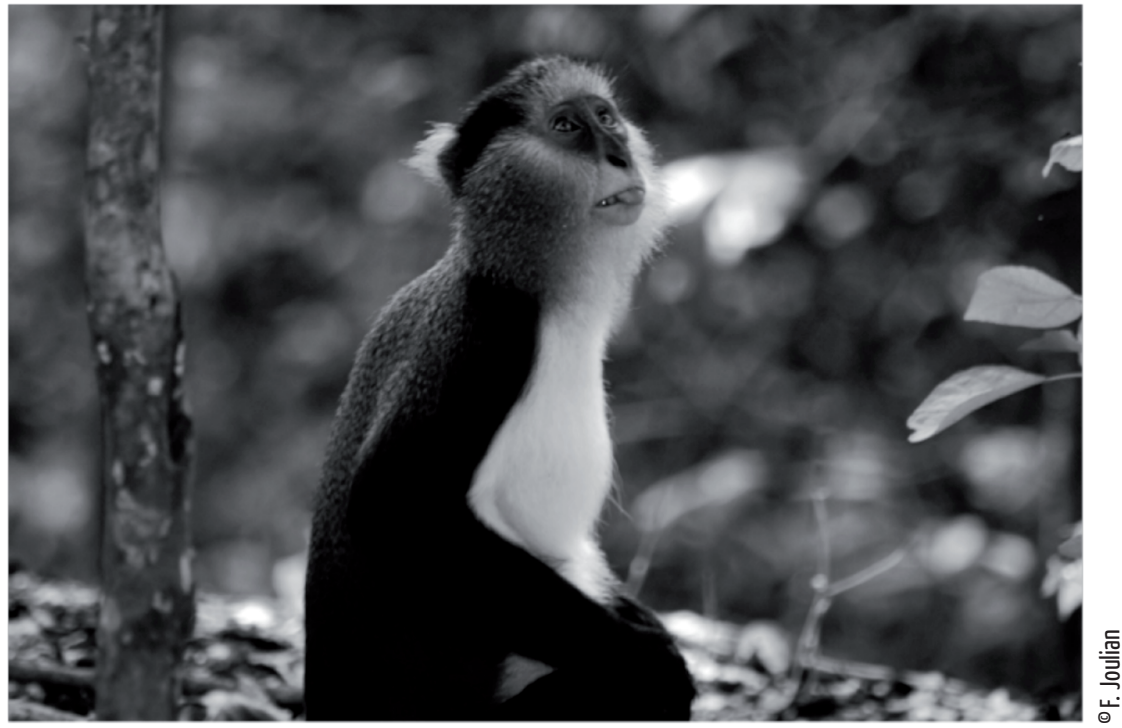

Dès notre arrivée, nous notons cependant les contrastes entre un grand respect envers les primates et des activités de chasse omniprésentes aux alentours (Joulian, Abbeg 2008).

Là encore, l'ethnologue s'interroge sur la diversité des pratiques et des instruments qui peuvent, dans le cas présent, davantage blesser le chasseur que le gibier!

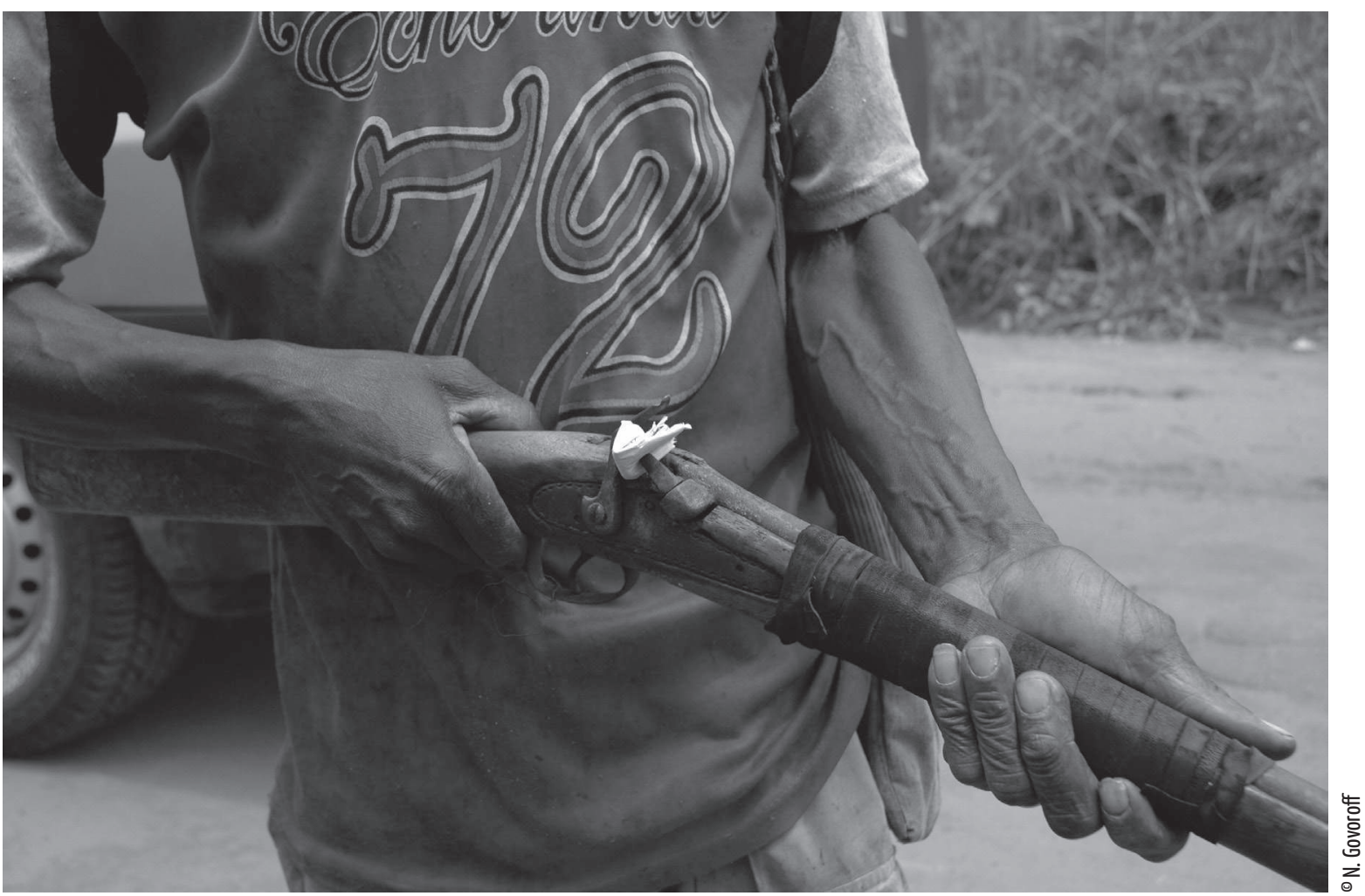




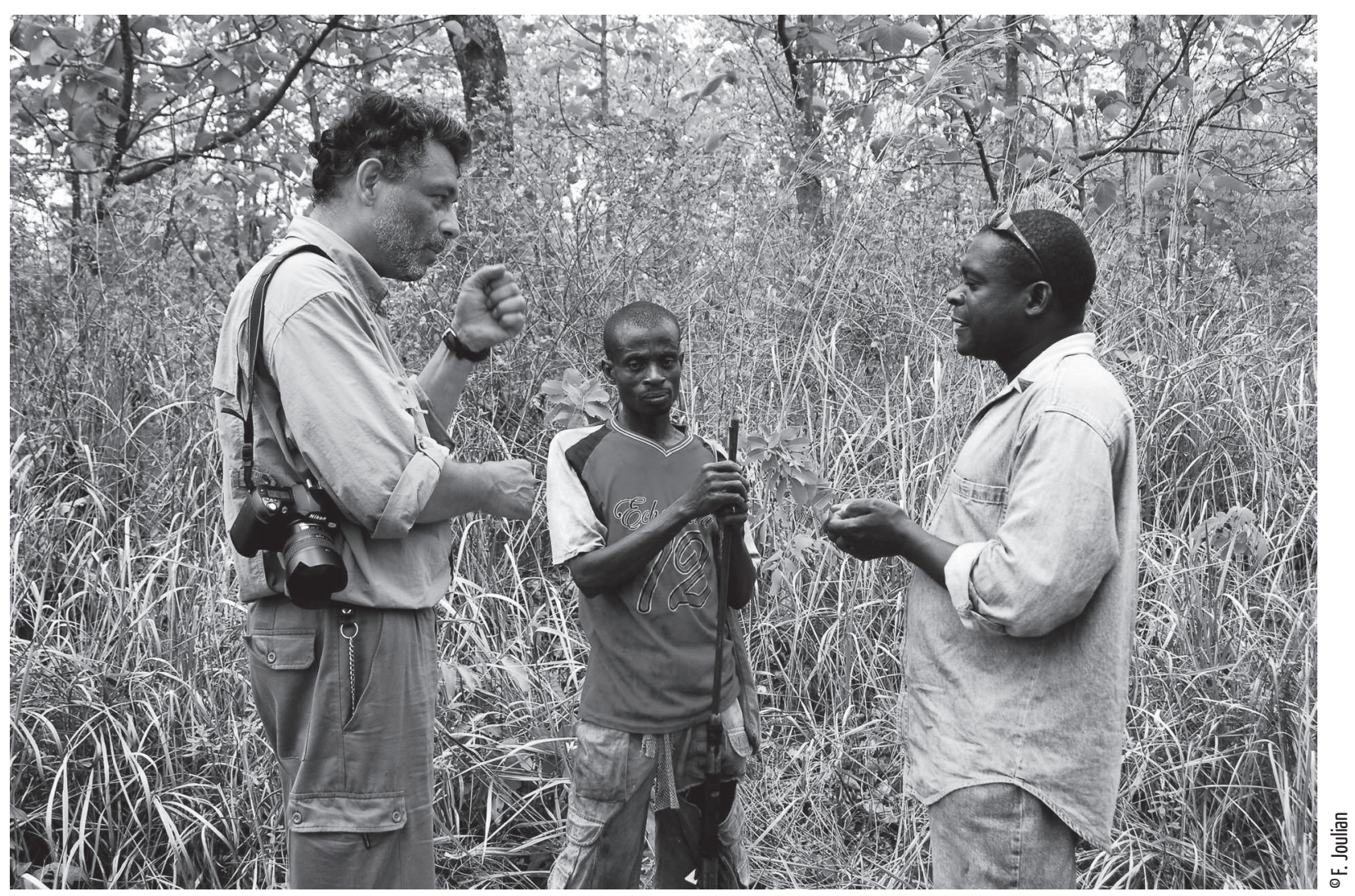

« Parler technique » : l'ethnologue, l'enquêté (dubitatif), l'interprète et... le photographe Boabeng-Fiema, Ghana, 2007

Enseigne « Buffalo » sur la porte d'entrée d'une armurerie-quincaillerie Téchiman, Ghana 2007

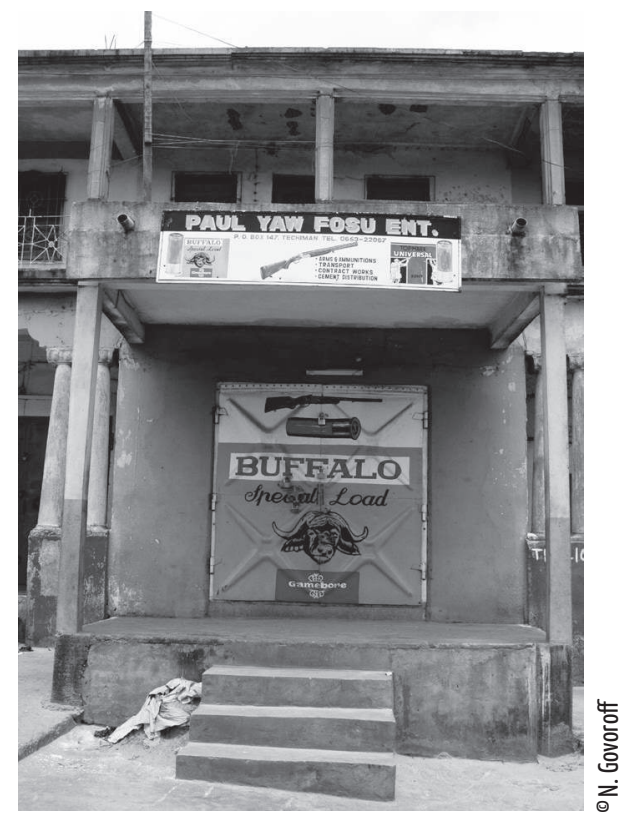


1. Certains auteurs considèrent que les armes traditionnelles sont plus efficaces que les fusils, par exemple Ross (1978). Pour la thèse opposée, voir Hames et Vickers (eds) 1983.

2. Quelques jeunes le font en remplaçant les plombs par des poids de pêche en plomb de $4 \mathrm{~mm}$ (ou plus) de diamètre. La chaleur produite par le feu les fond dans une masse compacte mais sans forme, peu utile à un chasseur. Tout d'abord, parce que l'aérodynamique du projectile rend la trajectoire imprévisible au-delà d'une certaine distance (courte) puis, comme leur usage implique une visée précise, le chasseur perd le bénéfice de l'augmentation d'énergie d'impact.

3. La portée utile est la distance jusqu'à laquelle un projectile est mortel et n'a besoin d'aucune correction de visée. Parmi d'autres paramètres, la longueur du canon est déterminante. Les experts balistiques français considèrent que la portée maximum utile est atteinte avec des tubes longs de $75 \mathrm{~cm}$. Les Américains, toutefois, arrivent à une conclusion différente et produisent des canons d'un mètre de long pour la chasse à l'oie (Noblet 1988).

4. Un « poste » est un lieu où le gibier risque plus probablement de passer et où les chasseurs l'attendent. Le terme est utilisé aussi pour les affûts à grives.
5. Le chevreuil, introduit il y a une dizaine d'années, n'est pas prisé contrairement à ce que sa taille aurait pu laisser présager.

6. Tout le grand gibier mâle est castré. On peut faire remonter cette pratique au XIV ${ }^{\mathrm{e}}$ siècle (Fabre-Vassas $1982: 383)$

7. La castration des mâles morts n'est jamais faite par des femmes, mais le plus souvent par leur mari qui est d'habitude posté pas très loin.

8. Les lecteurs gauchers sauront par expérience ce qu'on veut dire ici.

9. Une comparaison avec la taille du calibre recommandé par les experts nationaux (Noblet 1988) montre que les chasseurs de la vallée du Verdon utilisent massivement des plombs plus gros que nécessaire.

10. L'usage de chevrotines a depuis été interdit par la loi en France.

11. Jusqu'à un certain point, les balles trouvées par terre peuvent aussi être décodées, mais parce que leur présence n'est pas intentionnelle, elles sont simplement des marques, et non des signes.

\section{RÉFÉRENCES}

Bromberger, C. 1979 Technologie et analyse sémantique des objets: pour une sémio-technologie. L'Homme 19 (1): 105-40.

Bromberger, C et Dufour, A. H. 1982 Pourquoi braconner? Jeux interdits en Basse-Provence. Études rurales 87-8 (La Chasse et la cueillette aujourd'hui): 357-75.

Bromberger \& al. 1981 Les Paysans varois et leurs collines: les enjeux symboliques d'une passion. In Forêt méditerranéenne III (1) : 45-55.

— 1985 À la recherche des pratiques et usages quotidiens en Provence : guide d'enquêtes ethnographiques. Marseille: Centre national de documentation pédagogique.

Chamboredon, J.-C. 1982 La Diffusion de la chasse et la transformation des usages sociaux de l'espace rural. Études rurales 87-8: 233-60.

Fabre-Vassas, C. 1982 Le Partage du férum. Un rite de chasse au sanglier, Études rurales 87-88: 377-400.

Geistdoerfer, A. 1973 Leroi-Gourhan: Méthode d'analyse des techniques, La Pensée 171 : 60-74.

Govoroff, N. C. 1985 Espaces de chasse dans la haute vallée du Verdon, Mémoire de Maîtrise en ethnologie, Université de Paris X-Nanterre.

— 1987 Un rituel de chasse au sanglier en Haute-Provence, Atelier 9 : 29-33.

Hames, R. B. et Vickers, W. T. (sous la direction de) 1983 Adaptive Responses of Native Amazonians. New York: Academic Press.

Ingold, T. 1980 Hunters, Pastoralists and Ranchers. Cambridge: Cambridge University Press (2 édition). 
Leroi-Gourhan, A. 1971 (1943) L'Homme et la Matière. Paris: Albin Michel.

— 1973 (1945) Milieu et techniques. Paris: Albin Michel.

Mauss, M. 1983 (1936) Les Techniques du corps. In Sociologie et anthropologie. Paris: Presses universitaires de France : $365-86$

Noblet, N. 1988 Le Permis de chasser. Paris: Hachette (11édition).

Ross, E. 1978 Food taboos, diet and hunting strategy; the adaptation to animals in Amazon cultural ecology, Current Anthropology 19 (1): 1-36.

Tabet, P. 1979 Les Mains, les outils, les armes, L'Homme 19 (3-4): 5-63.

Traimond, B. 1984 Le Braconnage du gibier dans la Lande, XIX-Xx ${ }^{e}$ siècle; dits et interdits, Ethnologie française 14 (4): 355-62.

Turnbull, C. 1965 Wayward Servants: the two worlds of the African pygmies. Garden City: National History Press.

— 1973 The importance of flux in two hunting societies. In R. Lee et I. DeVore (dir.) Man the Hunter. Chicago : Aldine (4édition) : 132-37.

\section{RÉFÉRENCES DE L'INTRODUCTION}

Govoroff, N. 2000 Le Geste et les paroles : l'ethnologue et l'apprenti. In Quels langages pour les techniques? (21 janvier 2000). Journées 1999-2000. «État des recherches sur les pratiques et représentations de la technique » F. Joulian, S. de Cheveigné, P. Geslin (org.) Paris : Collège de France.

— 2007 Looking for Chimps. A Social Anthropologist's Point-of-view. In : Workshop "The anthropology of relationships between humans and primates" European Federation for Primatology, Prague. http:// userweb.pedf.cuni.cz/kbio/efp/abstract.php

— 2007b Des Plaisirs de la chaîne opératoire. In Techniques et sociétés (8 février 2007). Journées scientifiques dans le cadre du séminaire « Éléments d'anthropologie fondamentale : objets, techniques et cultures » F. Joulian, O. Gosselain (org.). Paris : Musée du Quai Branly.

— s/p Les Koulango de l'Ouest et les animaux. In Hommes et primates en perspective. F. Joulian (dir.). Paris : Éditions de la Maison des sciences de l'homme (à paraître).

Guyonnet, M.-H. 2008 Bricolage des savoirs et quête de prestige : la chasse au poste en Provence. In Bromberger et Guyonnet (dir.) De la nature sauvage à la domestication de l'espace. Enquêtes ethnologiques en Provence et ailleurs. Hommage à Annie-Hélène Dufour. Aix-en Provence : Presses Universitaires de Provence : 119-134.

Joulian, F. Abegg, C. 2008 Zoos et cause animale. Perspectives éthologique et anthropologique. In : Techniques \& culture 50 « Les Natures de l'Homme » : 120-143.

Joulian, F. 1995 Compte rendu de « Technological Choices : Transformation in Material Cultures since the Neolithic. Pierre Lemonnier (ed.) 1993 London \& New York : Routledge, 420 p. ». Études Rurales 133$134: 199-201$.

Lemonnier, P. (ed.) 1993 Technological Choices : Transformation in Material Cultures since the Neolithic. London $\&$ New York : Routledge, $420 \mathrm{p}$.

Testart, A., Govoroff, N., Lécrivain, V. 2002 Les Prestations matrimoniales. L’Homme 161 : 165-196. 